\title{
Design of Window based FIR filter for Electrocardiogram Noise Removal
}

\author{
Ameer Rasel.V.B, A.Uma,P.Kalpana ${ }^{1}$ \\ \{arvb.ece@psgtech.ac.in, au.ece@psgtech.ac.in,pk.ece@psgtech.ac.in\} \\ Student, ME VLSI Design, PSG College of Technology ${ }^{1}$, Assistant Professor (Sel.Grade), \\ PSG College of Technology ${ }^{2}$, Professor, PSG College of Technology ${ }^{3}$
}

\begin{abstract}
Cardiovascular diseases are the major threat for the survival of human being. Electrocardiogramis used in the diagnosis of the heart diseases. But many types of noises are present to deter the quality of ECG signals. Thus, these noises must be filtered using Digital filter like FIR (Finite Impulse Response)filter.There are different approaches to design a FIR filter. One of the prominent ways is by usingwindows.There are other ways of designing the FIR filters but filtering using windows is one of the fastest ways of designing the FIR filters. In this paper different orders to filter has been analyzed and the best order to filter the ECG is found. Using that order different windows of FIR filter has been designed using Xilinx System Generator which is used to filter the noisy ECG signal. Also, various windows have been compared for filtering noises in ECG like Base line Wander noise and Muscle Artifacts noise. The best window among all the window is found out using MATLAB Simulink.
\end{abstract}

Keywords: Finite Impulse Response (FIR), Electrocardiogram (ECG), Muscle Artifacts (MA), Baseline Wander (BW).

\section{Introduction}

Signal Processing [1] is a branch of Electrical Engineering which uses Mathematics as a tool to do the analysis, synthesis and modification of information carrying signals like audio, video etc.Signal processing has its application found in a wide variety of fields like biomedical engineering, acoustics, control system, seismology, process control, genomics etc. Out of all the signal processing techniques the most commonly used technique is filtering.Filtering [2] in signal processing is elimination or attenuation of the unwanted signals like interference and noises from the signal to attain the signal of interest.

Heart is the most crucial and essential part of the human body and its proper functioningis mandatory for the survival of human beings. There are many deadly diseases associated with heart called Cardiovascular diseases like High blood pressure, Cardiac arrest, Congestive heart failure, Arrhythmia, Stroke etc. Careful observation of the functioning of the heart is essential for the diagnosis of the above diseases. Thus,Electrocardiography is essential which is the study of the electrical activities of heart for a period, using electrodes on skin. These electrodes are used to detect small beats on the skin because of expansion and contraction of heart. Typically, ECG signal frequency ranges from $0.5 \mathrm{~Hz}$ to $100 \mathrm{~Hz}$ and is as shown in fig 1 . 


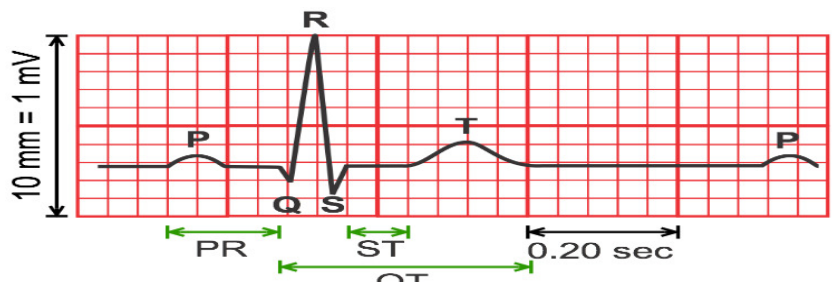

Fig 1.ECG of a heart in normal sinus rhythm

There are three components of ECG: The P wave [3], which represents the depolarization of the atria; the QRS complex, which represents the depolarization of the ventricles; and the T wave, which represents the repolarization of the ventricles.

Noises in Electrocardiogram

As is the case of any signals ECG also containing noises associated with it. There are various noises associated with ECG like:

1.Baseline Wander [4] is a low frequency noise which is mainly due to the patient movements mainly breathing.

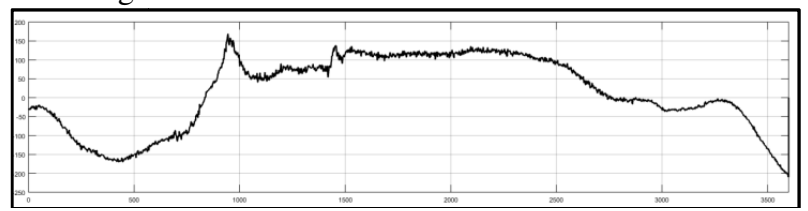

Fig 2: Baseline wander noise

2.Electromagnetic Interference [5] is caused by the interference of the other electronic equipment like Air conditioner, Mobile Phones etc. whose frequencywill be around Radio frequency.

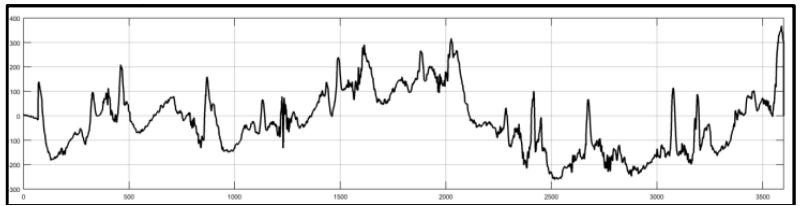

Fig 3. Electromagnetic interference noise

3.Muscle artifacts noise is due to the movement of muscle patient due to cold or shivering of the patient.

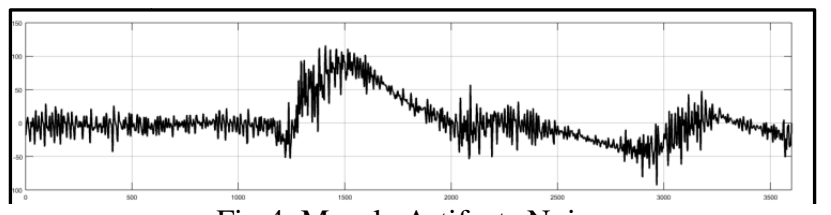

.Fig 4. Muscle Artifacts Noise

\section{Filter design using windows}

Windowing technique [6] is one of the quickest and the simplest way of designing FIR filter. Filter designed using windowing technique approaches ideal filters with some approximation errors. Designing the ideal low pass filter in the frequency domain with a cut 
off frequency Fc and transforming this to time domain results in sinc function that extends to infinity in both side, which results in substantial number of taps which is not practical thus it must be truncated. To do sothe impulse response has been multiplied with a window function which truncate the infinite impulse response to a finite impulse which is needed by a FIR filter This is done using windows. Different windows used in FIR filter design are:

1.Rectangular window is the simplest window, but it has small stop band attenuation for same number of taps, it limits the impulse response of the filter by multiplying it with a constant window function and its range will also be specified by the windowing function ineq (1)

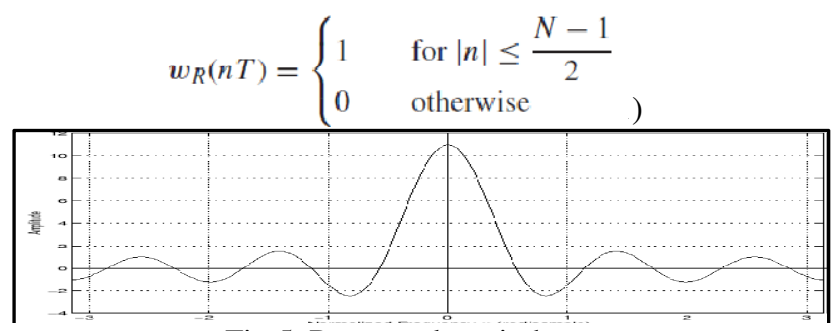

Fig 5. Rectangular window

2. Kaiser window is used to control theside lobes using the main lobes by varying the parameters. It tries to maximize the energy concentration at main lobes. It gives the information of the amount of ripple and the transition bandwidth than the filter length. The width of the main lobe is controlled the filter length.Its window function is given in eq (2)

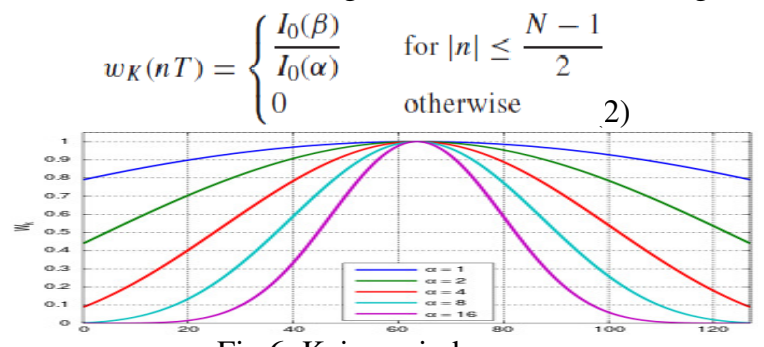

Fig 6. Kaiser window

3.Hamming Windowis used to effectively make the side lobes to just touch the zero, it is also called as raised cosine transform and its window function is given in eq (3)

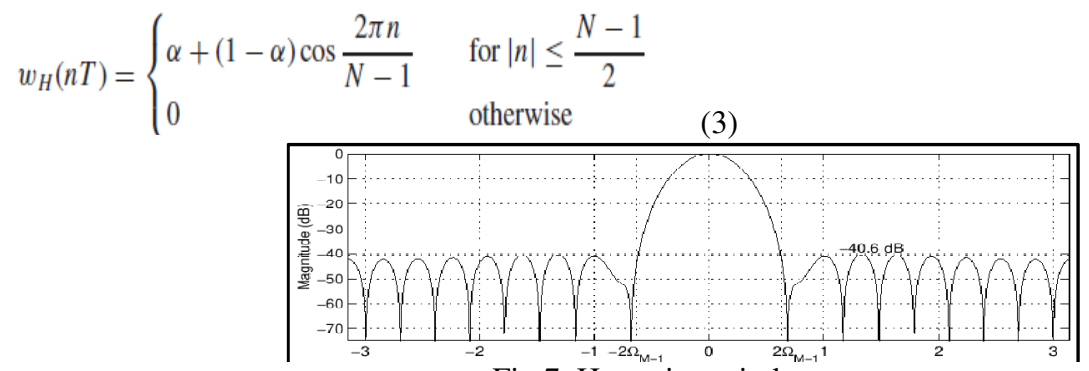

Fig 7. Hamming window

4.Blackmann Window very much like the hamming window except the minor changes in the window function, to reduce the ripples also called Gibbs oscillation and its window function is given in eq (4) 


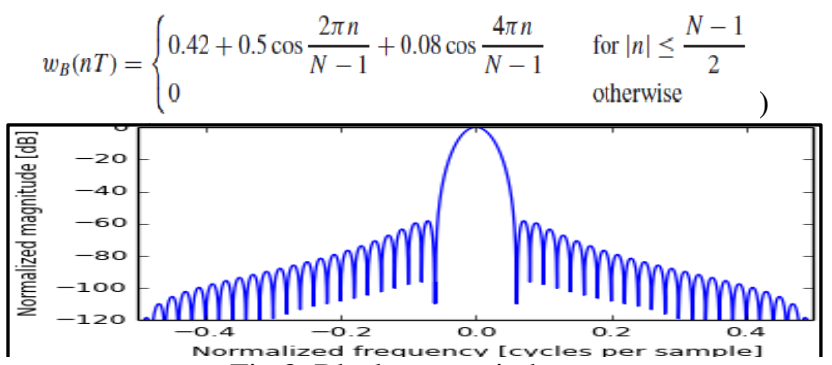

Fig 8. Blackmann window

5.Bartlett window is very similar to triangle function except that the first and last sample of the window are always zero, and its window function is given in eq (5)

$$
w(n)= \begin{cases}\frac{2 n}{N}, & 0 \leq n \leq \frac{N}{2} \\ 2-\frac{2 n}{N}, & \frac{N}{2} \leq n \leq N\end{cases}
$$

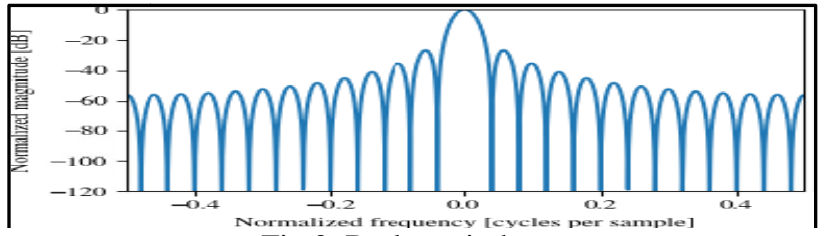

Fig 9. Barlett window

\section{ECG Filtering Using FDA Tool}

Initial Simulation was done with ECG signal from MIT-BIH ECG Database in Physionet.org [7] and taking band limited white noise as noise for ECG signal from Simulink library.

ECG data 106 sample has been imported to MATLAB workspace using the Signal from workspace block which is added with Band limited Noise and is given

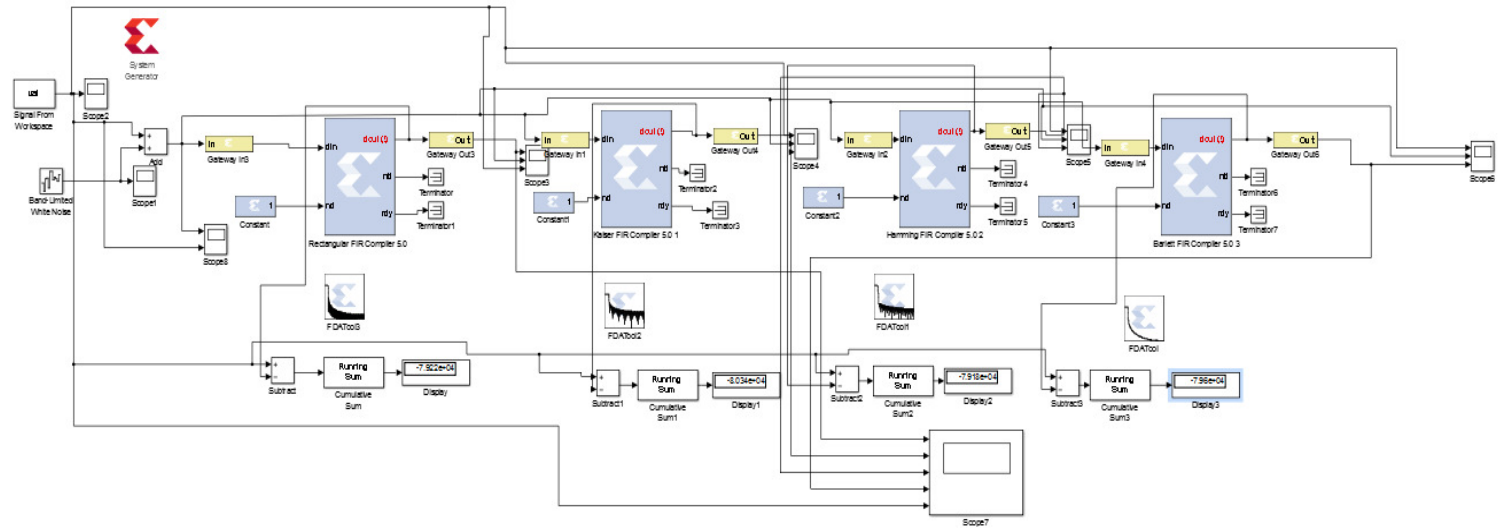

Fig 10. Filter Setup with 4 windows 
as input to the FIR filter [8] designed using FDA Tool [9] for different windows.

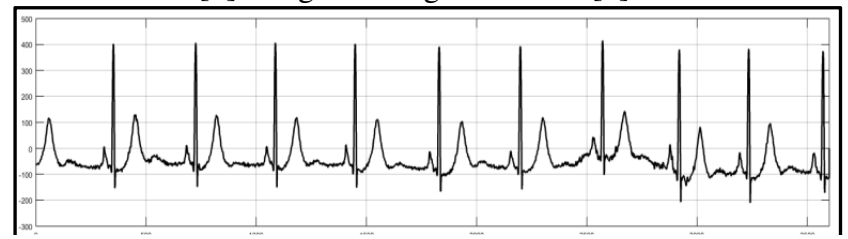

Fig 11. 106 ECG sample

After the signal has been added with the noise it looks like

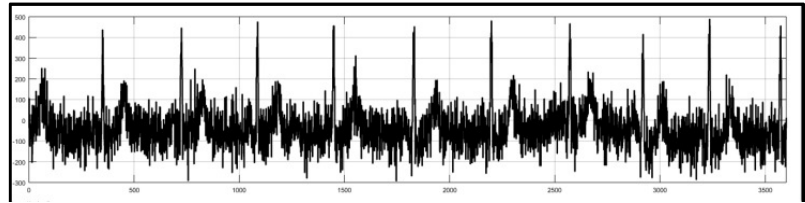

Fig 12. Noisy Signal

After filtering the noisy signal using 4 windows, the output waveform looks like
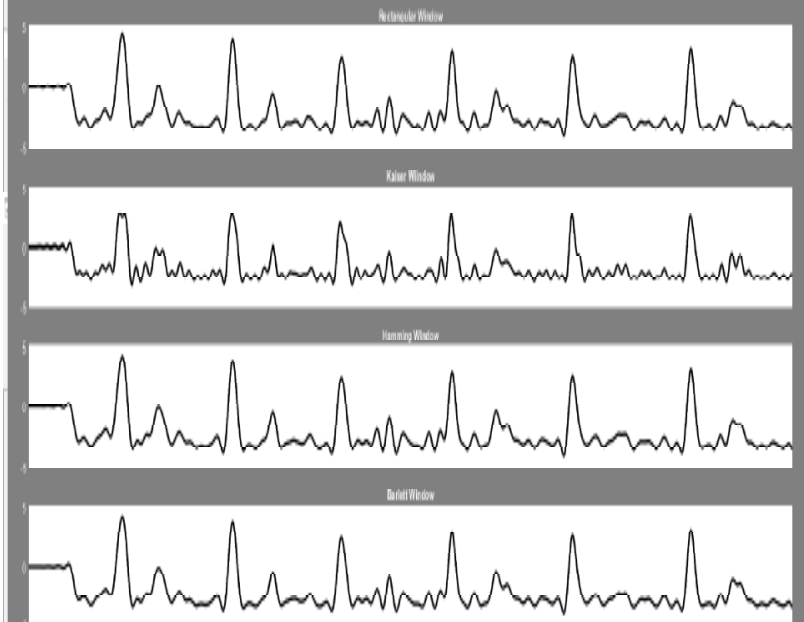

Fig 13. Filtered signal using 4 windows

TABLE I Analysis Of Different Windows

\begin{tabular}{|l|l|l|}
\hline S.No & Window & Error \\
\hline 1. & Rectangular & 1.223 \\
\hline 2. & Kaiser & 1.222 \\
\hline 3. & Hamming & 1.223 \\
\hline 4. & Barlett & 1.223 \\
\hline
\end{tabular}

On the analysis Kaiser windows is found to be the best based on their error among all four windows. 


\section{Simulation Results}

Real time noises like Muscle artifacts noise and Baseline wander noise are taken from the Phsionet.org website, it has been imported to MATLABworkspace [10]andhas been added with original ECG signal. Noisy signal has beenapplied to the FIR filter designed using different windows.

Two parameters have been used to analyze the performance namely SNR and Error.

1.Signal to Noise Ratio [11] is defined as the Ratio of Signal to the Noise of the Filtered signal, and it is given as:

Output SNR $=20 \times \log 10($ VarianceofSOVarianceof $(\boldsymbol{S O}-\boldsymbol{S f}))$

whereSO is the Original Signal

Sf is the Filtered Signal

2. Error is another parameter used to analyze the performance of the filter and it is just the difference between the filtered output and the original input.

Error $=$ Filtered Output - Original Input

The Simulink model for the filtering of the noise has been given in the following figure 12

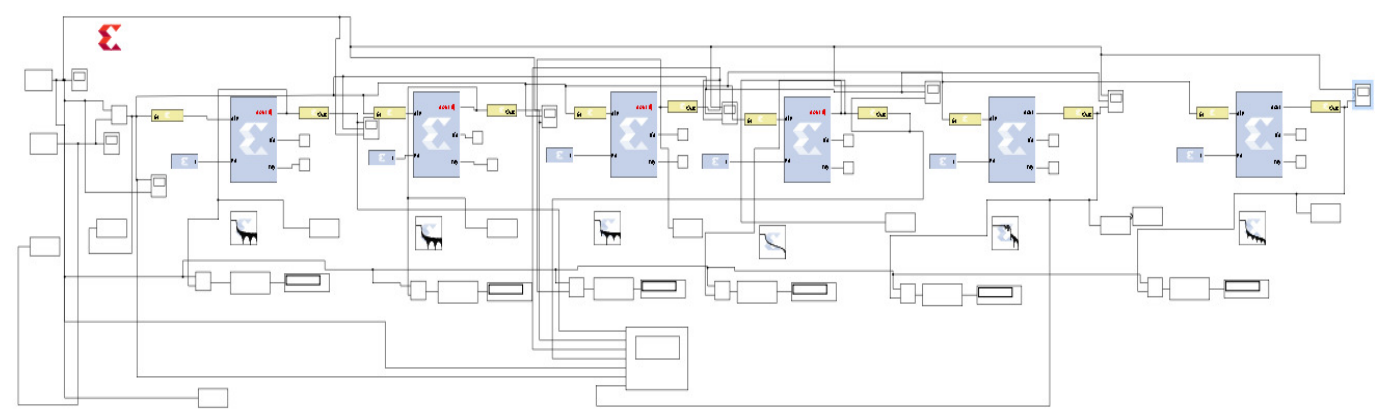

Fig 14. Simulink Model for MA Noise filtering

ECG ANALYSIS WITH MA NOISE WITH DIFFERENT WINDOWS

TABLE II Setting The Order Of The Filter

\begin{tabular}{|l|l|l|l|}
\hline Order & Error & SNR & Waveform \\
\hline 25 & 5.282 & 0.0048 & Improved \\
\hline 50 & 5.284 & 0.0050 & Improved \\
\hline 75 & 5.294 & 0.0056 & Improved with delay \\
\hline 100 & 5.304 & 0.0058 & No improvement \\
\hline $\mathbf{1 2 5}$ & $\mathbf{5 . 2 9 8}$ & $\mathbf{0 . 0 0 6 4}$ & Major improvement \\
\hline 150 & 5.303 & 0.0066 & Improved with delay \\
\hline
\end{tabular}

For the filtering of MA Noise [12], 102 sample has been taken from Physionet.org and it has been added with the MA Noise and the noisy signal is given as input to all the filter designed with various windows. 


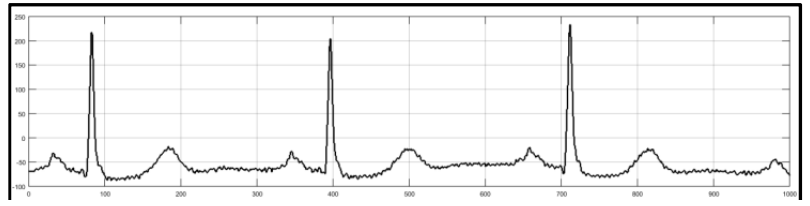

Fig 15. 102 sample

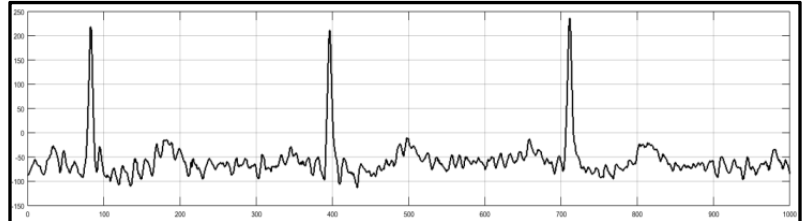

Fig 16. 102 sample added with Muscle Artifacts Noise

The outputs of FIR filters designed using different windows [13] is given in the Fig 15.

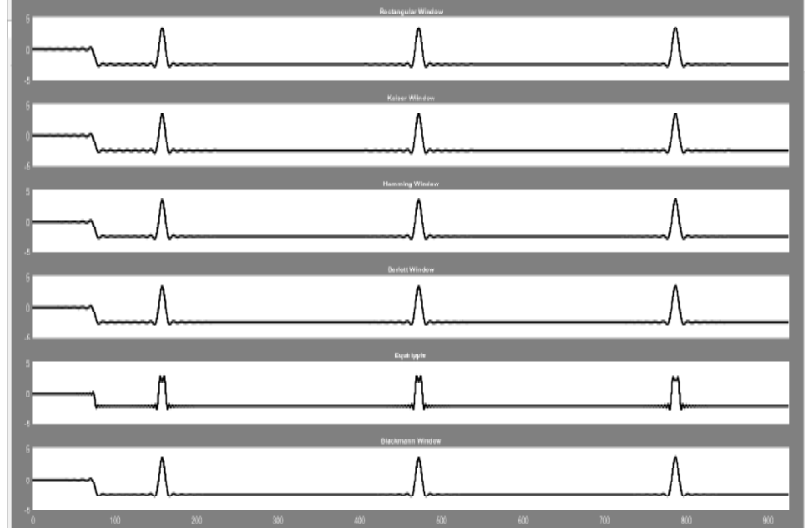

Fig 17. Output of various windows for MA Noise filtering

TABLE III Analysis of Various Filters In Ma Noise Filtering

\begin{tabular}{|l|l|l|l|l|}
\hline Window & Input SNR & Output SNR & Error & Delay \\
\hline Rectangular & -0.8879 & 0.0064 & 5.298 & 71 \\
\hline Kaiser & $\mathbf{- 0 . 8 8 7 9}$ & $\mathbf{0 . 0 0 6 4}$ & $\mathbf{5 . 2 9 8}$ & $\mathbf{7 0}$ \\
\hline Hamming & -0.8879 & 0.0064 & 5.299 & 71 \\
\hline Barlett & -0.8879 & 0.0062 & 5.301 & 70 \\
\hline Equi-ripple & -0.8879 & 0.0164 & 5.170 & 73 \\
\hline Blackman & -0.8879 & 0.0064 & 5.299 & 70 \\
\hline
\end{tabular}

Thus, it can be concluded that the Kaiser is the best windows based on their SNR, Error and Delay.

ECG ANALYSIS WITH BW NOISE WITH DIFFERENT WINDOWS

The same procedure has been repeated for BW Noise [14 \& 15] and the analysis has been done and the results are found out to be 


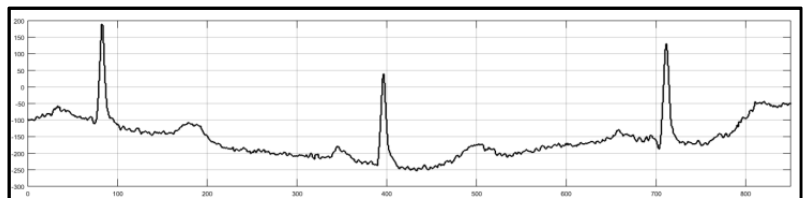

Fig 18. 102 sample added with Baseline Wander Noise

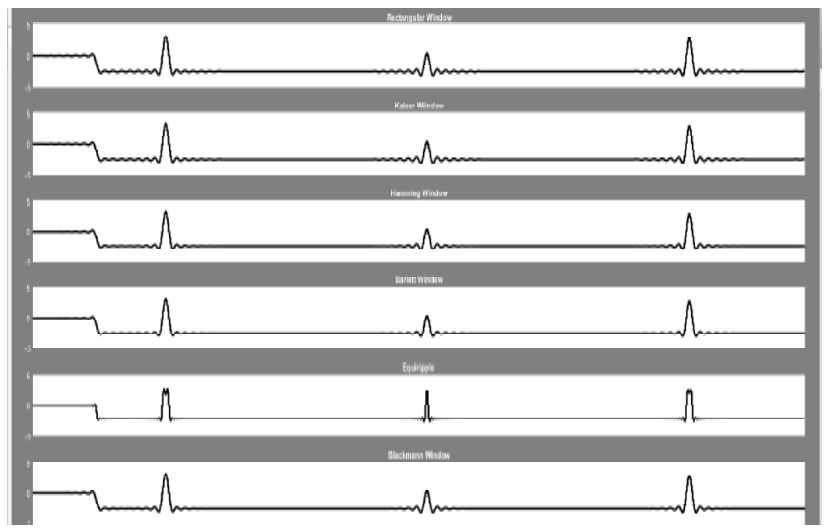

Fig 19. Output of various windows for BW Noise filtering

TABLE IV Analysis Of Various Filters In BW Noise Filtering

\begin{tabular}{|l|l|l|l|l|}
\hline Window & Input SNR & Output SNR & Error & Delay \\
\hline Rectangular & -12.1447 & 0.0014 & 4.820 & 71 \\
\hline Kaiser & $\mathbf{- 1 2 . 1 4 4 7}$ & $\mathbf{0 . 0 0 1 4}$ & $\mathbf{4 . 8 2 0}$ & $\mathbf{7 0}$ \\
\hline Hamming & -12.1447 & 0.0014 & 4.821 & 71 \\
\hline Barlett & -12.1447 & 0.0014 & 4.823 & 70 \\
\hline Equi-ripple & -12.1447 & 0.0038 & 4.703 & 73 \\
\hline Blackmann & -12.1447 & 0.0014 & 4.821 & 70 \\
\hline
\end{tabular}

Again, like the case of MA Noise it is found that the Kaiser window is the best window based on their SNR, Error and Delay.

\section{Conclusion}

ECG is a recorded plot of the heart's electrical activity. It gives crucial information about the heart's proper functioning, but this ECG is deterred by several noises from various sources like Muscle Artifact Noise, Baseline Wander Noise. To denoise the ECG signals FIR filter has been designed using various windowing techniques. FIR filter designed using various windows is compared and their performance has been analyzed,the best order of the filter for the reliable filtering of the ECG signal has been found out to be 125 and the performance of various windows has been compared. Comparison shows that the Kaiser window stands the best among all the other windows in case of FIR filter. 


\section{References}

[1] A. V. Oppenheim, and R. W. Schafer, "Discrete-Time Signal Processing”, Englewood Cliffs, NJ: Prentice Hall. ISBN-10: 0131988425, Issue: 1989

[2] Beylkin, G.; Lewis, R.D.; Monzon, L.- On the Design of Highly Accurate and Efficient IIR and FIR Filters, Signal Processing, IEEE Transactions on Volume: 60, Issue: 8,2012.

[3] http://en.wikipedia.org/wiki/Electrocardiogram.

[4] Tripti Singh1, Prabhakar Agarwal2, Dr. V.K Pandey3 "ECG Baseline noise removal techniques using window-based FIR filters" 2014 International Conference on Medical Imaging, m-Health and Emerging Communication Systems (MedCom)

[5] Chavan M. S., Agarwala R., and Uplane M.D., Suppression of Baseline Wander and power line interference in ECG using Digital IIR Filter'International Journal of Circuits, Systems and Signal Processing, issue 2, volume 2,2008

[6] Eleti, A.A,Zerek, A.R. - FIR digital filter design by using windows method with MATLAB, IEEE 978-1-4799- 2953-52013, Issue: 12,2013

[7] www.physionet.org.

[8] GaoweiXu, Yao Zou, Jun Han, Xiaoyang Zeng "Low Power Design for FIR Filter" State Key Laboratory of ASIC and System, Fudan University, Shanghai 200433, China.

[9] https://users.encs.concordia.ca/ blynch/elec442lab5.pdf

[10] http://www mathworks.com/

[11] https://stackoverflow.com/questions/26618318/matlab-mean-square-error-vs-snr-plot

[12] P.C. Bhaskar, M.D.Uplane, "High Frequency Electromyogarm Noise Removal from Electrocardiogram using FIR low pass filter based on FPGA", ELSEVIER Procedia technology 25 (2016) 497-504.

[13] S. Haykin and B. Widrow, "Least-Mean-Square Adaptive Filters", Hoboken, NJ, USA: Wiley, ISBN10: 0471215708 Issue: 2003.

[14] Digvijay J. Pawar and P. C. Bhaskar "FPGA Based FIR Filter Design for enhancement of ECG Signal by MinimizingBase-line Drift Interference" International Journal of Current Engineering and Technology ISSN 2277 - 4106,Vol.3, No.5 (December 2013).

[15] Ms. GeetaKadam andProfPCBhaskar,"Reduction of Power Line Interference in ECG Signal Using Fir Filter” IJCER I Mar-Apr 2012 I Vol. 2 | Issue No.2 1314-319 